\title{
On outindependent subgraphs of strongly regular graphs
}

\author{
M. A. FIOL* and E. GARRIGA \\ Departament de Matemàtica Aplicada IV. Universitat Politècnica de Catalunya. \\ Jordi Girona 1-3. Mòdul C3. Campus Nord, 08034 Barcelona. Spain
}

Communicated by W. Watkins

(Received 25 March 2005)

\begin{abstract}
An outindependent subgraph of a graph $\Gamma$. with respect to an independent vertex subset $\mathrm{C} \subset \mathrm{V}$. is the subgraph $\Gamma_{C}$ induced by the vertices in $r \backslash C$. We study the case when $\Gamma$ is strongly regular. where the results of de Caen [1998. The spectrat of complementary subgraphs in a strongly regular graph. European Journal of Combinatorics. 19(5). 559 565.]. allow us to derive the whole spectrum of $\Gamma_{C}$. Moreover, when $C$ attains the Hoffman Lovasz bound for the independence number. $\Gamma_{C}$ is a regular graph (in fact. distance-regular if $\Gamma$ is a Moore graph). This article is mainly devoted to study the non-regular case. As a main result. we characterize the structure of $\Gamma_{C}$ when $C$ is the neighborhood of either one vertex or one edge.
\end{abstract}

Kentords: Strongly regular graph: Independent set; Spectrum

AMS Subject Classifications: 05C50:05E30

\section{Preliminaries}

In this article we address the following question: Let $\Gamma=(V, E)$ be a strongly regular graph with a given independent set $C \subset V$. What can be said about the structure of the graph $\Gamma_{C}$ induced by the vertices in $V \backslash C$ ? The study of $\Gamma_{C}$, which we call the outindependent graph, can be motivated, for instance, by the study of some possible distance-regular graphs, such as the Moore graph with degree 57 and diameter 2. Moreover. this work could also have some relevance to the study of completely regular codes. Some known results (see [1.2]), allow us to compute the whole spectrum of $\Gamma_{C}$. and prove that it is a regular graph precisely when the cardinality of $C$ attains the Hoffman-Lovász (spectral) bound for the independence number. In fact when such a bound is attained in a Moore graph, the corresponding outindependent graph turns out to be a distance-regular graph. A known example of this fact occurs when

*Corresponding author. Email: fiol a mat.upc.es

Limear and Mulrilinear Alechra

ISSN 0308-1087 print: ISSN 1563-5139 online C 2005 Taylor \& Francis Group LId http: www.tandf.co.uk journals 
$\Gamma=H S$ (the Hoffman-Singleton graph) and $C$ is a maximum independent set with 15 vertices, in which case $\Gamma_{C}=O_{4}$ (the 'odd graph' [3] with degree 4 ); see Jeurissen [4]. The main results of this article, contained in section 3, deal with the case when $\Gamma_{C}$ in a non-regular graph. In particular, some cases where the non-trivial component of $\Gamma_{C}$ is a distance-regular graph are characterized. In the rest of this introductory section. we summarize some of the background used in our study.

\subsection{Graphs and their spectra}

Consider the adjacency matrix $\boldsymbol{A}$ of a regular graph $\Gamma=(V, E)$ on $n=|V|$ vertices, with spectrum

$$
\operatorname{sp} \Gamma:=\operatorname{sp} \boldsymbol{A}=\left\{\lambda_{0}^{m_{0}}, \lambda_{1}^{m_{1}} \ldots, \lambda_{d}^{m_{d}}\right\}
$$

where the eigenvalues $\lambda_{i}, 0 \leq i \leq d$, are in decreasing order, $\lambda_{0}>\lambda_{1}>\cdots>\lambda_{d}$, and the superscripts denote multiplicities. The set of such eigenvalues is denoted by ev $\Gamma$. and their corresponding eigenspaces are $\mathcal{E}_{i}:=\operatorname{Ker}\left(\boldsymbol{A}-\lambda_{i} \boldsymbol{I}\right), 0 \leq i \leq d$. The orthogonal projections onto the eigenspaces $\mathcal{E}_{i}$ are represented by the so-called (principal) idempotents of $\boldsymbol{A}$ :

$$
E_{i}:=\frac{1}{\phi_{i}} \prod_{j=0(j \neq i)}^{d}\left(A-\lambda_{j} I\right) \quad(0 \leq i \leq d)
$$

where $\phi_{i}:=\prod_{i=0(j \neq i)}^{d}\left(\lambda_{i}-\lambda_{j}\right)$. Given any subset $C$ with $r:=|C| \geq 1$ vertices, we consider its normalized characteristic vector $\boldsymbol{e}_{C}:=(1 / \sqrt{r}) \sum_{u \in C} \boldsymbol{e}_{u}$. where $\boldsymbol{e}_{u}$ denotes the $u$-th standard unit basis vector in $\mathbb{R}^{\prime \prime}$. Then. the $C$-multiplicity of the eigenvalue $\lambda_{i}$ is defined by

$$
m_{C}\left(\lambda_{i}\right):=\left\|\boldsymbol{E}_{i} \boldsymbol{e}_{C}\right\|^{2}=\left\langle\boldsymbol{E}_{i} \boldsymbol{e}_{C}, \boldsymbol{e}_{C}\right\rangle=\frac{1}{r} \sum_{u, v \in C}\left(\boldsymbol{E}_{i}\right)_{t r} \quad(0 \leq i \leq d) .
$$

Note that, since $\boldsymbol{e}_{C}$ is a unit vector, we have $\sum_{i=0}^{d} m_{C}\left(\lambda_{i}\right)=1$. Moreover, we see from the above that, if $\Gamma$ is connected, the $C$-multiplicity of $\lambda_{0}$ is $m_{C}\left(\lambda_{0}\right)=r / n>0$. In particular, when $C$ is a single vertex $u$, the $\{u\}$-multiplicities of $\lambda_{i}$ correspond to the so-called "(local) $u$-multiplicities" (see [5]). If $\mu_{0}\left(=\lambda_{0}\right)>\mu_{1}>\cdots>\mu_{d c}$ represent the eigenvalues in ev $\Gamma$ with non-zero $C$-multiplicities, the (local) $C$-spectrum of $C$ is

$$
\mathrm{spC}:=\left\{\mu_{0}^{m_{n_{1}}}>\mu_{1}^{\tilde{m}_{1}}>\cdots>\mu_{d_{c}}^{\tilde{m}_{d_{C}}}\right\}
$$

where $\tilde{m}_{i}:=m_{C}\left(\mu_{i}\right), 0 \leq i \leq d_{C}$, and $d_{C}(\leq d)$ is called the dual degree of $C$. It is known that - if $\Gamma$ is connected, then the eccentricity of $C$. defined by $\operatorname{ecc}(C):=\max _{u \in I}$. $\operatorname{dist}(u, C)=\max _{u \in r} \min _{r \in C} \operatorname{dist}(u, v)$, is bounded above by $d_{C}$. (For more details, see $[6,7]$.)

The following theorem is usually known as "the interlacing theorem" (see, for instance [2.8]). 
THEOREM 1.1 Let $\Gamma$ be a graph with $n$ vertices and eigenvalues $\theta_{1} \geq \theta_{2} \geq \cdots \geq \theta_{n}$. Let $\Gamma^{\prime}$ an induced subgraph of $\Gamma$. with $m<n$ vertices and eigenvalues $\xi_{1} \geq \xi_{2} \geq \cdots \geq \xi_{m}$. Then the eigenvalues of $\Gamma^{\prime}$ interlace the eigenvalues of $\Gamma$; that is.

$$
\theta_{i} \geq \xi_{i} \geq \theta_{n-m+i} \quad(1 \leq i \leq m)
$$

In particular, if $\Gamma^{\prime}$ is an independent set of $m$ vertices then $\xi_{i}=0$ for any $i=1, \ldots, m$, and the above gives $\theta_{m} \geq 0 \geq \theta_{n-m+1}$. Hence. the independence number of $\Gamma$. denoted by $\alpha(\Gamma)$, always satisfies

$$
\alpha(\Gamma) \leq \min \left\{\left|\left\{i: \theta_{i} \geq 0\right\}\right| .\left|\left\{i: \theta_{i} \leq 0\right\}\right|\right\} .
$$

This bound is due to Cvetkovic [9]. (For a discussion of this and other interesting applications of interlacing. we refer the reader to Haemers [2].)

\subsection{Characterizing distance-regularity}

Let $C \subset V$ be a vertex subset of a regular connected graph $\Gamma$, with $\operatorname{ecc}(C)=\varepsilon$. Then, we say that $\Gamma$ is distance-regular around $C$ if the distance partition $V=C_{0} \cup C_{1} \cup \cdots \cup C_{\varepsilon}$. where $C_{i}=\Gamma_{i}(C):=|\{u: \operatorname{dist}(u, C)=i\}|$, is regular; that is, the numbers

$$
c_{i}(u):=\left|\Gamma(u) \cap C_{i-1}\right|, \quad a_{i}(u):=\left|\Gamma(u) \cap C_{i}\right|, \quad b_{i}(u):=\left|\Gamma(u) \cap C_{i+1}\right|
$$

where $u \in C_{k}, 0 \leq i \leq \varepsilon$, depend only on the values of the distance $i$, but not on the chose vertex $u$. Then, the set $C$ is also referred to as a completely regular set or completely regular code. In [6], the authors obtained the following characterization of distance-regularity around a set $C$, in terms of its local spectrum and the number of vertices at maximum possible distance from $C$.

THEOREM 1.2 Let $\Gamma=(V, E)$ be a regular comnected graph. A vertex subset $C \subset V$, with $r$ vertices and local spectrum sp $C=\left\{\mu_{0}^{m_{0}}>\mu_{1}^{m_{1}}>\cdots>\mu_{d_{0}}^{m_{i c}}\right\}$. is a completely regular code if and only if the number $n_{d_{c}}:=\left|C_{d_{C}}\right|$ of vertices at distance $d_{C}$ from $C$ is

$$
n_{d_{C}}=r\left(\sum_{i=0}^{d_{\mathrm{c}}} \frac{\tilde{m}_{0}^{2} \tilde{\pi}_{0}^{2}}{\tilde{m}_{i} \tilde{\pi}_{i}^{2}}\right)^{-1}
$$

where $\tilde{\pi}_{i}:=\prod_{i=0(j \neq i)}^{d_{C}}\left|\mu_{i}-\mu_{j}\right|, 0 \leq i \leq d_{C}$.

Moreover, when $C$ consists of a single vertex $u$ and the above condition holds for any $u$, with $\tilde{m}_{i}$ replaced by $m_{i} / n, 0 \leq i \leq d$, the authors [10] showed that we get a 'quasi-spectral' characterization of distance-regular graphs:

THEOREM 1.3 A regular connected graph $\Gamma=(V, E)$, with $n$ vertices and spectrum $\mathrm{sp} \Gamma=\left\{\lambda_{0}^{m_{0}}>\lambda_{1}^{m_{1}}>\cdots>\lambda_{d}^{m_{l}}\right\}$, is distance-regular if and only if the number $n_{d}(u)$ of vertices at distance $d$ from every vertex $u \in V$ is

$$
n_{d i}(u)=n\left(\sum_{i=0}^{d} \frac{\pi_{0}^{2}}{m_{i} \pi_{i}^{2}}\right)^{-1}
$$

where $\pi_{i}:=\prod_{i=0(j \neq i)}^{d i}\left|\lambda_{i}-\lambda_{j}\right|, 0 \leq i \leq d$. 
The corresponding result for antipodal distance-regular graphs was proved in [11]: THEOREM 1.4 A regular connected graph $\Gamma$ on $n$ vertices, with eigenvalues $\lambda_{0}>$ $\lambda_{1}>\cdots>\lambda_{d}$, is a r-antipodal distance-regular graph if and only if the distance graph $\Gamma_{d}$ is constituted by disjoint copies of the complete graph $K_{r}$, with

$$
r=2 n\left(\sum_{i=0}^{d} \frac{\pi_{0}}{\pi_{i}}\right)^{-1}
$$

where the $\pi_{i}$ 's are defined as above.

\subsection{Strongly regular graphs}

Recall that a (not necessarily connected) graph $\Gamma=(V, E) \neq K_{n}$ is $(n, k ; a, c)$-strongly. regular if $\Gamma$ is $k$-regular, with $n$ vertices, and every pair of adjacent (respectively, nonadjacent) vertices have $a$ (respectively, $c$ ) common neighbors. For the basic theory of strongly regular graphs we refer the reader to Cameron [12] or Godsil [13]. Note that $\Gamma$ is connected if and only if $c \neq 0$. In this case. which will be assumed hereafter, $\Gamma$ is a distance-regular graph with diameter $d=2$ and intersection array $\iota(\Gamma)=$ $\{k . k-a-1: 1, c\}$. Consequently, the adjacency matrix $A$ of $\Gamma$ satisfies the equation

$$
\boldsymbol{A}^{2}-(a-c) \boldsymbol{A}-(k-c) \boldsymbol{I}=c \boldsymbol{J}
$$

whence the eigenvalues of $\Gamma$ are $\lambda_{0}=k$ and the zeros of the (Hoffman) polynomial $H=(1 / c)\left[x^{2}-(a-c) x-(k-c)\right]($ see $[14])$ :

$$
\theta, \tau=(a-c \pm \sqrt{\Delta}) / 2 \text { where } \Delta:=(a-c)^{2}+4(k-c)
$$

being $\theta \geq 0$ and $\tau<0$. (For a strongly regular graph we follow Godsil's notation [13] and let $\theta, \tau$ stand for $\lambda_{1}, \lambda_{2}$, respectively.) Moreover, recall that a graph $\Gamma$ with adjacency matrix $\boldsymbol{A}$ and diameter $d$ is distance-regular if and only if there exists a sequence of (orthogonal) polynomials $\left(p_{i}\right)_{0 \leq i \leq l}$, dgr $p_{i}=i$, such that the distance- $i$ matrix $\boldsymbol{A}_{i}$ (that is, the adjacency matrix of the distance graph $\Gamma_{i}=\left(V, E_{i}\right)$ where $u v \in E_{i}$ if and only if $(u, v)=i$ in $\left.\Gamma\right)$ is given by $p_{i}(\boldsymbol{A})$. Then the multiplicities of the eigenvalues of $\Gamma$ or, alternatively, the values of the polynomial $p_{d}$ at $\mathrm{ev} \Gamma$, are given by the formulae

$$
m_{i}=(-1)^{i} \frac{\pi_{0} p_{d}\left(\lambda_{0}\right)}{\pi_{i} p_{d}\left(\lambda_{i}\right)}, \quad p_{d}\left(\lambda_{i}\right)=(-1)^{i} \frac{\pi_{0}}{\pi_{i}} \frac{p_{d}\left(\lambda_{0}\right)}{m_{i}} \quad(0 \leq i \leq d)
$$

where $\pi_{i}:=\prod_{i=0(j \neq i)}^{d}\left|\lambda_{i}-\lambda_{j}\right|$; see, for instance, Bannai and Ito [15]. Since for a $(n, k ; a, c)$-strongly regular graph we have $p_{0}=1, p_{1}=x, p_{2}=(1 / c)\left(x^{2}-a x-k\right)$. and $n=H(k)=1+k+p_{2}(k)$, the above gives $m_{0}=1$ (as expected. since $\Gamma$ is connected) and

$$
\begin{aligned}
& m_{1}=\frac{-\tau(n-1)-k}{\theta-\tau}=\frac{1}{2}\left(n-1-\frac{2 k+(n-1)(a-c)}{\sqrt{\Delta}}\right) . \\
& m_{2}=\frac{\theta(n-1)+k}{\theta-\tau}=\frac{1}{2}\left(n-1+\frac{2 k+(n-1)(a-c)}{\sqrt{\Delta}}\right) .
\end{aligned}
$$


(Note that, in this case, $m_{1}$ and $m_{2}$ can also be derived as the solution of the system with equations $m_{1}+m_{2}=n-1$ and $m_{1} \theta+m_{2} \tau=-k$.)

Conversely, any regular graph with three distinct eigenvalues $k>\theta>\tau$ is known to be a $(n, k ; a, c)$-strongly regular graph with parameters

$$
n=\frac{(k-\theta)(k-\tau)}{k+\theta \tau}, \quad a=k+\theta+\tau+\theta \tau, \quad c=k+\theta \tau .
$$

The following discussion allows us to exclude some extreme cases from our study. Let us first consider the value of $k-\theta+\tau=k-\sqrt{\Delta}$. For a given value of $k \geq 3$ (the case $k \leq 2$ being trivial), the function $\Delta=\Delta(a, c)=(a-c)^{2}+4(k-c)$, defined in $[0, k-2] \times[1, k]$, has absolute maximum $\Delta(0, k)=k^{2}$, and also $\Delta(0,1)=9$ in the case $k=3$, and absolute minimum $\Delta(k-2, k)=4$. Thus,

$$
0 \leq k-\theta+\tau \leq k-2
$$

Moreover. equality on the left occurs when $\Gamma$ has parameters $(2 k, k ; 0, k)$, which corresponds to the complete bipartite graph $K_{k, k}$ (with eigenvalues $k, 0,-k$ ), and in the particular case $(10,3 ; 0,1)$ of the Petersen graph $P$ (with eigenvalues $3,1,-2)$; and equality on the right is attained for the parameters $(k+2, k ; k-2, k)$, corresponding to the "cocktail-party graph' $C P_{k}$ (with eigenvalues $k, 0,-2$ ): see Biggs [3]. In any other case. the strongly regular graphs considered here have eigenvalues satisfying $2<\theta-\tau<k$. Notice that both $K_{k, k}$ and $C P_{k}$ are instances of complete multipartite graphs which, as discussed below, will also be removed as 'trivial cases.

A strongly regular graph $\Gamma$ is called imprimitive or trivial if either $\Gamma$ or its complement $\bar{\Gamma}$ (which is also a strongly regular graph) is not connected. It is known that $\Gamma$ is trivial if and only if $\Gamma=m K_{n}$ ( $m$ copies of the complete graph on $n$ vertices) or $\Gamma=K_{m(n)}$ (a multipartite complete graph with $m$ stable $n$-sets). While the first case has already been excluded since $c=0$, the second case occurs if and only if $c=k$ or, equivalently, $\theta=0$. Summarizing, henceforth it is always assumed that $\Gamma$ is a non-trivial strongly regular graph $(\theta>0, \theta \neq k)$ different from $P$.

\subsection{Independent sets in strongly regular graphs}

Let $\Gamma=(V, E)$ be a graph with diameter $d$. For a given integer $1 \leq h \leq d$, a vertex subset $C \subset V$ is said to be a $\Gamma_{h}$-clique (of order $r \geq 1$ ) if $C$ consists of $r$ vertices mutually at distance $h$. As a particular case of a more general result, we can find in [14] the following spectral characterization of a $\Gamma_{h}$-clique in a distance-regular graph.

PROPOSITION 1.5 Let $\Gamma$ be a distance-regular graph with $n$ vertices, diameter $d$, spectrum $\operatorname{sp} \Gamma=\left\{\lambda_{0}^{m_{0}}>\lambda_{1}^{m_{1}}>\cdots>\lambda_{d}^{m_{d}}\right\}$ and distance polynomials $\left(p_{h}\right)_{0 \leq h \leq d}$. For any $1 \leq h \leq d$, a subset $C \subset V$ with r vertices is a $\Gamma_{h}$-clique if and only if its $C$-multiplicities are given by.

$$
m_{C}\left(\lambda_{i}\right)=\frac{m_{i}}{n}\left(1+(r-1) \frac{p_{h}\left(\lambda_{i}\right)}{p_{h}\left(\lambda_{0}\right)}\right) \quad(0 \leq i \leq d) .
$$

Let us apply the above result to a (connected) $(n, k ; a, c)$-strongly regular graph $\Gamma$ with spectrum sp $\Gamma=\left\{k>\theta^{\prime \prime \prime}>\tau^{\prime \prime \prime}\right\}$ (no superscript means multiplicity one) given 
by (9). (11) and (12). Note first that, since such a graph has diameter 2, the maximum cardinality of a $\Gamma_{2}$-clique coincides with the maximum possible order of an independent set. Then, applying the well-known Hoffman-Lovász bound, hereafter denoted by $\mathrm{H}$, for the independence number $\alpha$ of a regular graph (see $[2,16]$ ) we get

$$
r \leq \alpha \leq \mathrm{H}:=\frac{n}{1-k / \tau}=\frac{\left(k^{2}+(c-a-1) k+c\right)(c-a+\sqrt{\Delta})}{c(2 k+c-a+\sqrt{\Delta})}
$$

where, as before, $\Delta=(a-c)^{2}+4(k-c)$. Other equivalent expressions for $\mathrm{H}$, obtained by using (13), are

$$
\mathrm{H}=\frac{-\tau}{c}(k-\theta)=\frac{\theta \tau-k \tau}{\theta \tau+k}=1-\frac{k}{c}(1+\tau)
$$

Now, by Proposition 1.5, the local multiplicities of an independent set $C$ of $\Gamma$, in terms of $r=|C|$ and $\mathrm{H}$, are

$$
\begin{aligned}
m_{C}(k) & =\frac{r}{n}>0, \\
m_{C}(\theta) & =\frac{m_{1}}{n}\left(1+(r-1) \frac{p_{2}(\theta)}{p_{2}(k)}\right)=\frac{m_{1}}{n}\left(1+(r-1) \frac{\pi_{0}}{\pi_{1} m_{1}}\right) \\
& =\frac{1}{n}\left(m_{1}+(r-1) \frac{k-\tau}{\theta-\tau}\right)=\frac{k-\tau}{\theta-\tau} \frac{H-r}{n} \geq 0 . \\
m_{C}(\tau) & =1-\frac{r}{n}-\frac{k-\tau}{\theta-\tau} \frac{H-r}{n}=\frac{k+\theta(n-1)}{n(\theta-\tau)}>0 .
\end{aligned}
$$

where we have used (10). Notice that, as expected, these numbers are non-negative. In fact, imposing such a condition to the first expression for $m_{C}(\theta)$ we get $r \leq 1-$ $\left(p_{2}(k) / p_{2}(\theta)\right)$. This can be shown to be equivalent, in our case of strong-regularity, to the Hoffman Lovász bound given by (15) (just use that $\left.p_{2}=(1 / c)\left(x^{2}-a x-k\right)\right)$.

\section{Outindependent graphs}

Let $C$ be an independent $r$-set of a strongly regular graph $\Gamma=(V, E)$ as above. Then, $C$ induces the partition $V=C_{0} \cup C_{1}$, where $C_{0}:=C$ and $C_{1}=V \backslash C$ (if $C$ is maximal; that is, $C$ is not a proper subset of any other independent set, then this partition is the 'distance partition' around $C$, and $\operatorname{ecc}(C)=1)$. In this section we derive the main results about the graph $\Gamma_{C}=\left(C_{1}, E_{1}\right)$, which we call the outindependent graph, induced by the vertices in $C_{1}$. To begin, notice that this graph has $\left|C_{1}\right|=n-r \geq n-r_{M}$ vertices, where $r_{M}$ represents the minimum of the bounds given in (4) and (15), and $\left|E_{1}\right|=|E|-k r=k(n / 2-r)$ edges. Moreover, since it is an induced subgraph, the following result is a direct consequence of the interlacing theorem.

LEMmA 2.1 Let $C$ be an independent r-set of a strongly regular graph $\Gamma$ with spectrum $\mathrm{sp} \Gamma=\left\{k>\theta^{m_{1}}>\tau^{m_{2}}\right\}$. Then, the eigenvalues of the outindependent graph $\Gamma_{C}$ are:

$$
(k \geq) \xi_{0} \geq \theta^{m_{1}-r} \geq \xi_{1} \geq \xi_{2} \geq \cdots \geq \xi_{r} \geq \tau^{m_{2}-r}
$$


where $r \leq \min \left\{m_{1}, m_{2}\right\}$. (Of course, a zero multiplicity means the absence of the corresponding eigenvalue.)

Proof Since $\Gamma$ is supposed to be non-trivial, we have that $\theta>0$ and hence Cvetkovic's bound (4) yields $r \leq m_{2}$. Thus, we only need to prove that $r \leq m_{1}$. But if we substitute $n=\mathrm{H}(1-k / \tau)$ into $(11)$, we get

$$
m_{1}=\frac{k-\tau}{\theta-\tau}(\mathrm{H}-1)>\mathrm{H}-1,
$$

whence $r \leq \mathrm{H}<m_{1}+1$ and we obtain the desired inequality, since $r$ is an integer.

From the above proof, note that always $r \leq r_{M}=\min \left\{m_{2}, H\right\}$. The following result shows that the regularity of $\Gamma_{C}$ only occurs when $|C|$ attains the Hoffman-Lovász bound, which consequently can only happen if $\mathrm{H} \leq m_{2}$. Using (12), (13) and (16), this condition is equivalent to

$$
k \geq \frac{-\tau(\theta-\tau)}{\theta+1}
$$

Proposition 2.2 Let $\Gamma=(V, E)$ be a strongly regular graph with spectrum $\mathrm{sp} \Gamma=$ $\left\{k>\theta^{m_{1}}>\tau^{m_{2}}\right\}$. Let $C \subset V$ be an independent set of $r$ vertices. Then the outindependent graph $\Gamma_{C}$ is regular if and only if $r=\mathrm{H}$. In this case. $\Gamma_{C}$ is connected and its degree is $\delta=k+\tau$.

Proof First note that any of the above statements imply that $C$ is a maximal set and hence $\operatorname{ecc}(C)=1$. Assume that $\Gamma_{C}$ is regular with degree $\delta$. Then $\Gamma$ is clearly distance-regular around $C$. In particular. $C$ has only two local eigenvalues and, by using the formulas (17). (18) and (19) for the local multiplicities, we conclude that $r=|C|=\mathrm{H}$. Conversely, assume now that $|C|$ attains the Hoffman-Lovász bound: $r=H$. Then. by the same formulas, its local spectrum turns out to be

$$
\operatorname{sp} C=\left\{\mu_{0}^{\tilde{m}_{0}}>\mu_{1}^{\tilde{m}_{1}}\right\}=\left\{k^{\mathrm{H} / n}>\tau^{1-(\mathrm{H} / n)}\right\}
$$

and notice that $r=\mathrm{H}$ is the unique value of $r$ for which $C$ has only two local eigenvalues. Then, noting that $\tilde{\pi}_{0}=\tilde{\pi}_{1}=\mu_{0}-\mu_{1}$, we get

$$
\mathrm{H}\left(\sum_{i=0}^{1} \frac{\tilde{m}_{0}^{2} \tilde{\pi}_{0}^{2}}{\tilde{m}_{i} \tilde{\pi}_{i}^{2}}\right)^{-1}=\mathrm{H}\left(\tilde{m}_{0}+\frac{\tilde{m}_{0}^{2}}{\tilde{m}_{1}}\right)^{-1}=\mathrm{H}\left(\frac{\mathrm{H}}{n}+\frac{\mathrm{H}^{2}}{n(n-\mathrm{H})}\right)^{-1}=n-\mathrm{H}
$$

so that, from Theorem 1.2, $\Gamma$ is distance-regular around $C$ and, in particular $\Gamma_{C}$ is regular of degree $\delta$, say. By counting the edges between $C$ and $V \backslash C$ we get $k \mathrm{H}=(k-\delta)(n-\mathrm{H})$, whence the degree $\delta$ of $\Gamma_{C}$ is as claimed. Finally, from $k>\theta-\tau$, we have, by using Lemma 2.1 , that $\xi_{0}(=\delta)>\theta \geq \xi_{1}$. Thus, $\delta$ has multiplicity one, which for a regular graph implies connectivity.

Most of this proposition can also be proved by using Haemers's method characterizing when the interlacing is 'tight' (see [2,17] for more details). 
Setting $s:=n-r$. with $r=|C|$. let $S$ denote the $s \times s$ adjacency matrix of the outindependent graph $\Gamma_{C}$. For proving the next result, which will allow us to derive the whole spectrum of $\Gamma_{C}$, we consider the vector $\boldsymbol{j}=(1,1, \ldots, 1) \in \mathbb{R}^{n}$ decomposed as $\left(\boldsymbol{j}_{0}, \boldsymbol{j}_{1}\right)$, with $\boldsymbol{j}_{0} \in \mathbb{R}^{r}$ and $\boldsymbol{j}_{1} \in \mathbb{R}^{\text {s. }}$.

Lemma 2.3 Let $C$ be an independent set with $r \geq 1$ vertices in an $(n, k ; a, c)$-strongly. regular graph $\Gamma=(V, E)$. Then the adjacency matrix $S$ of the outindependent graph $\Gamma_{C}$ satisfies the equation

$$
\left(\boldsymbol{S}^{2}-(k-c+a) \boldsymbol{S}\right) \boldsymbol{j}_{1}=-(k(a-c)+c r) \boldsymbol{j}_{1} .
$$

Proof Let $C_{0}=C$ and $C_{1}=V \backslash C$. With respect to the partition $V=C_{0} \cup C_{1}$, the adjacency matrix of $\Gamma$ and its square can be written as

$$
A_{3}=\left(\begin{array}{c|c}
\boldsymbol{O} & \boldsymbol{R} \\
\hline \boldsymbol{R}^{\top} & \boldsymbol{S}
\end{array}\right) . \quad \boldsymbol{A}^{2}=\left(\begin{array}{c|c}
\boldsymbol{R} \boldsymbol{R}^{\top} & \boldsymbol{R} \boldsymbol{S} \\
\hline \boldsymbol{S} \boldsymbol{R}^{\top} & \boldsymbol{R}^{\top} \boldsymbol{R}+\boldsymbol{S}^{2}
\end{array}\right)
$$

where $R$ is an $r \times(n-r)$ matrix corresponding to the adjacencies from $C_{0}$ to $C_{1}$. Then, from (8) we get

$$
\boldsymbol{R}^{\top} \boldsymbol{R}+\boldsymbol{S}^{2}-(a-c) \boldsymbol{S}-(k-c) \boldsymbol{I}=c \boldsymbol{J}
$$

whence the result follows by multiplying on the right by $j_{1}$ and using the facts that $\boldsymbol{R}_{1}=k \boldsymbol{j}_{0}, \boldsymbol{R}^{\top} \boldsymbol{j}_{0}=(k \boldsymbol{I}-\boldsymbol{S}) \boldsymbol{j}_{1}$. and $c(n-r)=k^{2}-(a-c) k-(k-c)-c r$.

From the above result we have that

$$
\begin{aligned}
\boldsymbol{j}_{1} & \in \operatorname{Ker}\left(\boldsymbol{S}^{2}-(k-c+a) \boldsymbol{S}+(k(a-c)+c r) \boldsymbol{I}\right) \\
& =\operatorname{Ker}(\boldsymbol{S}-\alpha \boldsymbol{I}) \oplus \operatorname{Ker}(\boldsymbol{S}-\beta \boldsymbol{I}),
\end{aligned}
$$

where $\alpha$ and $\beta$ are possible eigenvalues of $\Gamma_{C}$ given by

$$
\alpha \cdot \beta=\frac{1}{2}\left\{k+(a-c) \pm \sqrt{[k-(a-c)]^{2}-4 c r}\right\} .
$$

From these expressions, note that, since $r \geq 1$, we have $\alpha<k$ and $\beta>a-c=\theta+\tau$. In the following result we derive the whole ranges of variation of the possible values of $\alpha$ and $\beta$.

Lemma 2.4 Let $C$ be an independent set with $r \geq 1$ vertices in an $(n, k ; a, c)$-strongly. regular graph $\Gamma$ with eigenvalues $k>\theta>\tau$. Then the values of $\alpha$ and $\beta$ given above satisfy. the following inequalities:

$$
k>\alpha \geq k+\tau>\theta \geq \beta>\theta+\tau
$$


Proof Since it is always assumed that $k-\theta+\tau>0$. we only need to prove that $\alpha \geq k+\tau$ and $\beta \leq \theta$, and what happens in case of equality. For all this, consider the following simple computations, where we use again (15) and (16):

$$
\begin{aligned}
2 \alpha & =k+a-c+\sqrt{(k+a-c)^{2}-4 k(a-c)-4 c r} \\
& \geq k+\theta+\tau+\sqrt{(k+\theta+\tau)^{2}-4 k(\theta+\tau)-4 c H} \\
& =k+\theta+\tau+\sqrt{(k-\theta-\tau)^{2}+4 \tau(k-\theta)} \\
& =k+\theta+\tau+\sqrt{(k-\theta+\tau)^{2}}=2(k+\tau), \\
2 \beta & =k+a-c-\sqrt{(k+a-c)^{2}-4 k(a-c)-4 c r} \\
& \leq k+\theta+\tau-\sqrt{(k-\theta+\tau)^{2}}=2 \theta .
\end{aligned}
$$

Let $p$ be the monic polynomial generating the ideal $I:=\left\{q \in \mathbb{R}[x]: q(\boldsymbol{S})\left(\boldsymbol{j}_{1}\right)=0\right\}$. From the fact that $p$ divides the minimum polynomial of $\boldsymbol{S}$ and that $p_{C}:=(x-\alpha)(x-\beta)$ belongs to $I$. we have the following options for $p$ :

(a) $p=x-\alpha$ or $p=x-\beta$. Then $\boldsymbol{S}_{\boldsymbol{j}_{1}}=\alpha \boldsymbol{j}_{1}$ or $\boldsymbol{S}_{\boldsymbol{j}_{1}}=\beta \boldsymbol{j}_{1}$, and $\Gamma_{C}$ would be $\delta$-regular with degree $\alpha$ or $\beta$, respectively. But the second option is not possible since, otherwise, from Lemma 2.1 and (25) we would have $\beta=\xi_{0} \geq \theta \geq \beta$, so that $\beta=\theta$ : whereas, using Proposition 2.2, it should be $\delta=\beta=k+\tau>\theta$, a contradiction. Therefore it must be the first case, and $\Gamma_{C}$ is regular with degree $\alpha$. (Conversely, note that. if $\Gamma_{C}$ is regular. then the polynomial $p$ has degree one and, according to the above. $p=x-\alpha$.) Of course. this case coincides with the situation studied in Proposition 2.2, where $r=H$, and hence $\alpha=k+\tau$ is a simple eigenvalue.

(b) $p=(x-\alpha)(x-\beta)$. In this case, $\alpha$ and $\beta$ are eigenvalues of $\boldsymbol{S}, \Gamma_{C}$ is non-regular, $r<\mathrm{H}$ and. since $\alpha>\theta$. we have that $\xi_{0}=\alpha$ has also multiplicity one.

From this and Lemma 2.1, one can prove the following theorem, which is a direct consequence of the results of de Caen [1] relating the spectra of complementary induced
subgraphs in a strongly regular graph.

THEOREM 2.5 Let $C$ be an independent r-set of an (n.k;a,c)-strongly regular graph $\Gamma$ (not trivial and different from $P$ ). with corresponding outindependent graph $\Gamma_{C}$. Let $\alpha$
and $\beta$ be real numbers given by (24). Then.

$$
\operatorname{sp} \Gamma_{C}=\left\{\alpha>\theta^{m_{1}-r} \geq \beta>(\theta+\tau)^{r-i}>\tau^{m_{2}-r}\right\} .
$$

Here we discuss in a more detailed way the different possibilities, depending on the regularity and connectivity of $\Gamma_{C}$. The regular case (a) has been most studied and, basically. the new results presented here concern the non-regular case (b).

\subsection{The regular case}

Let us first assume that we have case (a). Then. $\Gamma_{C}$ is a connected regular graph with
spectrum

$$
\operatorname{sp} \Gamma_{C}=\left\{k+\tau>\theta^{m_{1}-\mathrm{H}+1}>(\theta+\tau)^{\mathrm{H}-1}>\tau^{m_{2}-\mathrm{H}_{1}}\right\} .
$$


As stated. this situation can only occur if $m_{2} \geq \mathrm{H}$. In particular. for those strongly regular graphs with $m_{2}=\mathrm{H}$ it turns out that the outindependent graph $\Gamma_{C}$, with $|C|=\mathrm{H}$, is again a strongly regular graph with spectrum

$$
\operatorname{sp} \Gamma_{C}=\left\{k+\tau>\theta^{m_{1}-\mathrm{H}+1}>(\theta+\tau)^{\mathrm{H}-1}\right\} .
$$

An interesting example of this situation is obtained when $\Gamma$ is the complement of the triangular graph $T(t):=L\left(K_{t}\right)$ (that is, the line graph of the complete graph on $t$ vertices: see [3]). Then the vertices of $\Gamma(t):=\overline{T(t)}$ correspond to the 2-subsets of the set $\{1,2 \ldots, t\}$, two vertices being adjacent when their corresponding subsets have void intersection. Thus $\Gamma(t)$ is a (strongly regular) graph on $n=\left(\begin{array}{l}t \\ 2\end{array}\right)$ vertices with spectrum

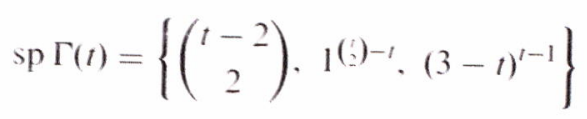

provided that $t \geq 4$. For instance, $\Gamma(4)$ is constituted by three copies of $K_{2}$ and $\Gamma(5)=P$. Thus, as we require our strongly regular graphs to be non-trivial and distinct from $P$, we assume hereafter that $t \geq 6$. Notice that $\Gamma(t)$ clearly has a maximum independent set $C$ with $\mathrm{H}=t-1$ vertices; just take $\{1, t\},\{2, t\}, \ldots,\{t-1, t\}$. Then, by (27) the spectrum of the corresponding outindependent graph is

$$
\begin{aligned}
\operatorname{sp} \Gamma(t)_{C} & =\left\{\left(\begin{array}{c}
t-2 \\
2
\end{array}\right)-t+3,1^{(t)-2 t+2},(4-t)^{t-2}\right\} \\
& =\left\{\left(\begin{array}{c}
t-3 \\
2
\end{array}\right), \mathrm{I}^{\left(\begin{array}{c}
(-1 \\
2
\end{array}\right)-t+1},(4-t)^{t-2}\right\}=\operatorname{sp} \Gamma(t-1) .
\end{aligned}
$$

as expected.

Let us see that the converse result also holds. First. any strongly regular graph $\Gamma$ whose Hoffman-Lovász and Cvetkovićs bounds coincide must have degree $k$ satisfying equality in (21). Moreover, if we assume that such a $\Gamma$ has an independent set $C$ attaining these bounds, and that the outindependent (strongly regular) graph $\Gamma_{C}$ has the same property as $\Gamma$ (that is, its Hoffman-Lovász bound equals that of Cvetković), it turns out that $\theta=1$. Then, taking into account that $\tau$ must also be an integer, we conclude that the spectrum of $\Gamma$ is as in (28). This means that the complement of $\Gamma$ has the same spectrum as the triangular graph $T(t)$. But it is known that triangular graphs are characterized by their spectrum, except for the case $t=8$ where there exist three more distinct graphs, $T^{\prime}(8), T^{\prime \prime}(8)$ and $T^{\prime \prime \prime}(8)$, cospectral with $T(8)$, called the Chang graphs: see [18-21]. (Obviously, the complements of all three Chang graphs have $\mathrm{H}=m_{2}=7$, as $\overline{T(8)}$ has, but the maximum independent set of $\overline{T^{\prime}(8)}$ consists only of 6 vertices, and the maximum independent sets of both $\overline{T^{\prime \prime}(8)}$ and $\overline{T^{\prime \prime}(8)}$ have only 5 vertices.) Summarizing, we arrive at the following conclusion:

PROPOSITION 2.6 The only infinite sequence of strongly. regular graphs with every "son' being an outindependent graph of its "father" are the complements of the triangular graphs.

The study of the general case $m_{2}>\mathrm{H}$ seems to be much more involved and, in general, the regular outindependent graphs $\Gamma_{C}$ are not distance-regular. In fact. we only know one case where this occurs. This is the case of Moore graphs; that is. 
the Petersen graph $P$, the Hoffman-Singleton graph $H S$, and the (possible) Moore graph with degree 57 and diameter 2, here denoted by $\Upsilon$. We will concentrate on the most interesting case $\Upsilon$, although the reasoning can also be applied to HS (whereas $P$ has already been excluded as a simple extreme case).

If it exists, our Moore graph $\Upsilon$ has intersection array $\iota(\Upsilon)=\{57,56 ; 1,1\}$, so that it is a strongly regular graph with parameters $a=0$ and $c=1$. Consequently, its eigenvalues are $k=57, \theta=7, \tau=-8$, and its order is $n=k^{2}+1=3250$. Its whole spectrum is $\operatorname{sp} \Upsilon=\left\{57,7^{1729},-8^{1520}\right\}$, and its distance polynomials are $p_{0}=1, p_{1}=x$, $p_{2}=r^{2}-57$. The Hoffman Lovász bound (15) yields now $r \leq \mathrm{H}=400$. Then, if $\Upsilon_{C}$ is non-regular $(1 \leq r<400)$, we conclude from Theorem 2.5 that it has spectrum

$$
\operatorname{sp} \Gamma_{C}=\left\{\alpha>7^{1729-r}>\beta>-1^{r-1}>-8^{1520-r}\right\}
$$

where. by (24), $\alpha=28+\sqrt{841-r} \in(49,57)$ and $\beta=28-\sqrt{841-r}$. (The above spectrum was also found by Schwenk [22] when $C$ is a subset of a vertex or edge neighborhood.) In this case, $\Upsilon_{C}$ is always connected (with diameter $3 \leq d \leq 4$ ). except for the two cases described in the following result.

Proposition 2.7 Let $C, r=|C|$, be an independent set of the Moore (57.2)-graph $\Upsilon=(V, E)$, with $1 \leq r<\mathrm{H}$. Then the outindependent graph $\Upsilon_{C}$ is non-connected if and only if either $C=\Gamma(u)$ or $C=\Gamma(u v)$, where $u \in V$ and $u v \in E$.

Proof From (32) and (40), we must look for an even integer $42 \leq h \leq 56$, such that $8 h$ divides $(h+58)(h+72)(h+42)$. So the only possible values are $h=54$ and $h=56$. with associated parameters

$$
\begin{array}{lllll}
\alpha=56 & \beta=0 & r=57 & n_{1}=3192 & n_{2}=1 \\
\alpha=55 & \beta=1 & r=112 & n_{1}=3136 & n_{2}=2 .
\end{array}
$$

corresponding to the two feasible options $C=\Gamma(u)$ and $C=\Gamma(u v)$, respectively.

A much more interesting consequence is obtained when $\Upsilon_{C}$ is regular. the case $|C|=\mathrm{H}$. In this case, the spectrum of $\Gamma_{C}$ is feasible for a distance-regular graph of diameter 3 and girth 5. Therefore, by a result of Brouwer and Haemers [23], we get the following proposition (which can also be proved from our characterization Theorem 1.3).

Proposition 2.8 When $C$ attains the Hoffman-Lozasz bound, the outindependent subgraph $\Upsilon_{C}$ of the possible Moore (57.2)-graph $\Upsilon$ is a distance-regular graph with order $n=2850$. degree $\delta=49$, diameter $d=3$, and intersection array

$$
\left.\iota \Upsilon_{C}\right)=\{49,48,8 ; 1,1,42\}
$$

As already mentioned. the same reasoning can be used to prove that the outindependent graph $H\left(S_{C}\right)$ of the Hoffman-Singleton graph $H S$, when $|C|=\mathrm{H}=15$, is the (distance-regular) odd graph $\mathrm{O}_{4}$. In the vein of the results of Theorem 3.1, we suspect that, in fact. the Moore graphs $\Upsilon$ and $H S$ are the only strongly regular graphs (with $\mathrm{H}<m_{2}$ ) whose regular outindependent graph is distance-regular. 


\section{The non-regular case}

Suppose now that we have case (b). Thus, $\Gamma_{C}$ is not regular. $1 \leq r<\mathrm{H}$ and, from (25). $\alpha>k+\tau>\theta>\beta$. Let $\Gamma_{C_{1}}, \Gamma_{C_{2}} \ldots \ldots \Gamma_{C_{i}}$ denote the connected components of $\Gamma_{C}$. Let $\boldsymbol{S}_{i}$ be the adjacency matrix of $\Gamma_{C}$ and $\boldsymbol{j}^{i}=(1, \ldots, 1) \in \mathbb{R}^{n_{i}}$ the part of $\boldsymbol{j}_{1}$ corresponding to $\Gamma_{C}, 1 \leq i \leq t$. Then the matrix $S$ can be written as a block diagonal matrix with blocks $S_{i}$, and the spectrum of $\boldsymbol{S}$ is the union of the spectra of the matrices $\boldsymbol{S}_{i}$. Then, considering that $\alpha$ and $\beta$ are simple eigenvalues, there exists a unique submatrix, say $S_{1}$, such that one of the two following subcases occurs:

(b1) $t=1$ and $\boldsymbol{j}^{\mathrm{i}} \in \operatorname{Ker}\left(\boldsymbol{S}_{1}-\alpha \boldsymbol{I}_{1}\right) \oplus \operatorname{Ker}\left(\boldsymbol{S}_{1}-\boldsymbol{\beta} \boldsymbol{I}_{1}\right)$. In this case, the outindependent graph $\Gamma_{C}=\Gamma_{C_{1}}$ turns out to be connected, and its spectrum is

$$
\operatorname{sp} \Gamma_{C}=\left\{\alpha>\theta^{m m_{1}-r}>\beta>(\theta+\tau)^{r-1}>\tau^{m_{2}-r}\right\} .
$$

(b2) $t=2$ and $\boldsymbol{j}^{i} \in \operatorname{Ker}\left(\boldsymbol{S}_{1}-\alpha \boldsymbol{I}_{1}\right), \dot{j}^{2} \in \operatorname{Ker}\left(\boldsymbol{S}_{i}-\boldsymbol{\beta} \boldsymbol{I}_{i}\right)$. Now $\Gamma_{C}$. with the same global spectrum as in (29), consists of two regular components, $\Gamma_{C_{1}}$ and $\Gamma_{C_{2}}$, with respective degrees $\alpha$ and $\beta$.

In what follows we analyze the possible situations in the second subcase. Let $\Gamma_{C_{1}}=\left(V_{1}, E_{2}\right)$ and $\Gamma_{C_{2}}=\left(V_{2}, E_{2}\right)$ the two regular components with respective degrees $\alpha>\beta(\alpha>\theta>\beta)$, which are the roots of the polynomial $p_{C}=x^{2}-(k+a-c) x+$ $k(a-c)+c r$. Since $\Delta^{\prime}=(k-a+c)^{2}-4 c r$ is the discriminant of such a polynomial. we see. from $\Delta^{\prime}=(\alpha-\beta)^{2}$, that $\Delta^{\prime}$ is a perfect square, say, $\Delta^{\prime}=h^{2}$, with $h$ a positive integer (with the same parity as $k+a-c=k+\theta+\tau$ ). This implies that the number of independent vertices must be of the form

$$
r=\frac{1}{4 c}\left\{(k-a+c)^{2}-h^{2}\right\}
$$

Let us write the involved parameters in terms of $k, \theta, \tau$ and $h$.

$$
\begin{aligned}
& \text { (13) } c=k+\theta \tau, \quad a=k+\theta+\tau+\theta \tau, \quad n=\frac{1}{c}(k-\theta)(k-\tau) ; \\
& \text { (24) } \alpha, \beta=\frac{1}{2}(k+\theta+\tau \pm h): \\
& \text { (30) } r=\frac{1}{4 c}(k-\theta-\tau+h)(k-\theta-\tau-h)=\frac{1}{c}(k-\alpha)(k-\beta) .
\end{aligned}
$$

Moreover. setting $n_{1}:=\left|V_{1}\right|$ and $n_{2}:=\left|V_{2}\right|$, the computation of these cardinalities and the number of edges between $C_{0}=C$ and $C_{1}=V \backslash C$ yields the linear system with unknowns $n_{1}$ and $n_{2}$ :

$$
\begin{gathered}
n_{1}+n_{2}=n-r \\
(k-\alpha) n_{1}+(k-\beta) n_{2}=r k
\end{gathered}
$$


with solutions

$$
\begin{aligned}
n_{1} & =\frac{1}{h}(-\beta(n-r)+k(n-2 r)) \\
& =\frac{1}{8 h c}(h+k-\theta-\tau)(h+k+\theta-\tau)(h+k-\theta+\tau) ; \\
n_{2} & =\frac{1}{h}(\alpha(n-r)-k(n-2 r)) \\
& =\frac{1}{8 h c}(h-k+\theta+\tau)(h-k+\theta-\tau)(h-k-\theta+\tau) .
\end{aligned}
$$

Furthermore, the spectrum

$$
\operatorname{sp} \Gamma_{C}=\left\{\alpha^{1}>\theta^{m_{1}-r}>\beta^{1}>(\theta+\tau)^{r-1}>\tau^{m_{2}-r}\right\}
$$

decomposes into

$$
\begin{aligned}
& \operatorname{sp} \Gamma_{C_{1}}=\left\{\alpha^{1}>\theta^{m_{1}-r}>(\theta+\tau)^{r-1-i}>\tau^{m_{2}-r-i}\right\} . \\
& \operatorname{sp} \Gamma_{C_{2}}=\left\{\beta^{1}>(\theta+\tau)^{i}>\tau^{i}\right\} .
\end{aligned}
$$

with $0 \leq i \leq r-1$ and $0 \leq i \leq m_{2}-r$.

Then. from the computation of the traces of $\boldsymbol{I}=\boldsymbol{S}_{2}^{0}, \boldsymbol{S}_{2}$ and $\boldsymbol{S}_{2}^{2}$, we get the following constraints for $i$ and $j$ :

$$
\begin{gathered}
i+j=n_{2}-1 \\
(\theta+\tau) i+\tau j=-\beta \\
(\theta+\tau)^{2} i+\tau^{2} j=\beta n_{2}-\beta^{2} .
\end{gathered}
$$

The compatibility of this linear system requires that

$$
h^{+}+\gamma_{3} h^{3}+\gamma_{2} h^{2}+\gamma_{1} h+\gamma_{0}=0 .
$$

where

$$
\begin{aligned}
\gamma_{3}:= & -2 \tau^{2}+2 \theta \tau . \\
\gamma_{2}:= & -2 \tau^{2}-2 k^{2}-2 \theta^{2} \tau-2 \theta^{2}+8 k \tau-2 \tau^{3}-2 k \theta \tau+6 k \tau^{2}+4 \theta \tau^{2}, \\
\gamma_{1}:= & 8 k \tau^{2}-2 \theta^{3} \tau-2 k^{2} \tau \theta-8 k^{2} \tau+4 k \theta^{2} \tau-2 \theta^{2} \tau^{2}+2 \tau^{4}+4 k \tau^{3}+2 \theta \tau^{3}-6 k^{2} \tau^{2}, \\
\gamma_{0}:= & 2 \tau^{5}+2 k^{3} \theta \tau-4 k^{2} \tau^{2} \theta-2 \theta^{2} k^{2} \tau+2 k \theta^{2} \tau^{2}+2 k \theta \tau^{3}-2 k \tau^{4}-2 \theta^{2} \tau^{2}-4 \theta^{2} \tau^{3} . \\
& \quad+k^{4}+2 k^{3} \tau^{2}+\theta^{4}+\tau^{4}-2 \theta^{2} k^{2}-2 k^{2} \tau^{2}-2 k^{2} \tau^{3}-2 \theta^{3} k \tau+2 \theta^{4} \tau .
\end{aligned}
$$

Moreover, the fourth degree polynomial (37) factorizes as

$$
\begin{aligned}
& (h-k+\theta+\tau)(h-k-\theta+\tau)\left(h^{2}-2\left(\tau^{2}-\theta \tau+\tau-k\right) h+2 \tau^{3}+\tau^{2}+2 k \tau+2 k \tau^{2}\right. \\
& \left.\quad-2 \theta^{2} \tau+k^{2}-\theta^{2}+2 k \theta \tau\right) .
\end{aligned}
$$


However. from (24), the root $k-\theta-\tau$ is not acceptable, since we would have $\alpha=k$ : neither is the root $k+\theta-\tau$ which would imply $\beta=\tau$ (note that. in both cases. (34) would give $n_{2}=0$ ). Consequently, the only possible values of $h$ are the integral roots of the polynomial

$$
Q:=h^{2}-2\left(\tau^{2}-\theta \tau+\tau-k\right) h+2 \tau^{3}+\tau^{2}+2 k \tau+2 k \tau^{2}-2 \theta^{2} \tau+k^{2}-\theta^{2}+2 k \theta \tau
$$

which satisfies

$$
Q(k-\theta+\tau)=4 c(k-\theta+\tau)>0 . \quad Q(-k-\theta-\tau-2 \theta \tau)=4 a \tau(\tau+1) \geq 0 .
$$

and has discriminant

$$
\Delta^{\prime \prime}:=4\left(\theta^{2} \tau^{2}-2 \theta \tau^{3}+\tau^{4}+2 \theta^{2} \tau-2 \theta \tau^{2}+\theta^{2}-4 k \tau^{2}-4 k \tau\right)
$$

Note that. from the definition of strong regularity, the degrees $\alpha, \beta$ must satisfy $k-\alpha \geq c, k-\beta \geq c, \alpha \geq a$ and $\beta \geq a$. Therefore.

$$
k>-\theta \tau=k-c \geq \alpha>\theta>\beta \geq a=k+\theta+\tau+\theta \tau \geq 0 .
$$

Translating these inequalities to constraints on $h$ we get

$$
k-\theta+\tau<h \leq-k-\theta-\tau-2 \theta \tau .
$$

With all the above. we now go back to (35) for studying the distinct cases depending on the number of different eigenvalues in $\operatorname{sp} \Gamma_{C_{z}}$.

Case $l$ Let $i=0, j=0$. Then $V_{2}$ is just the trivial graph with one vertex. $\beta=0$ and $n_{2}=1$. Therefore, $h=k+\theta+\tau-2 \beta=k+\theta+\tau$ and $\alpha=h+\beta=h$. The inequalities (40) become $k-\theta+\tau<k+\theta+\tau \leq-k-\theta-\tau-2 \theta \tau$, whence $(a=) k+\theta+\tau+$ $\theta \tau \leq 0$, that is $a=0$. The relevant parameters are:

$$
a=0 . \quad c=k-h . \quad r=k . \quad n_{1}=n-k-1 . \quad n_{2}=1 . \quad \alpha=h, \quad \beta=0 .
$$

Of course, if $V_{2}=\{u\}$, then the $r(=k)$ independent vertices are those adjacent to $u$. that is, $C=\Gamma(u)$. In this case we want to decide whether the connected component $\Gamma_{C_{1}}$ - induced by the vertex set $C_{1}=V \backslash(C \cup\{u\})$ - is a distance-regular graph or not. To this end. let $\Gamma$ have parameters $(n, k ; 0, c)$. Then. ev $\Gamma_{C_{\mathrm{i}}}=\left\{\mu_{0}>\right.$ $\left.\mu_{1}>\mu_{2}>\mu_{3}\right\}$ with

$$
\mu_{0}=k-c, \quad \mu_{2}=-c, \quad \mu_{1.3}=\theta, \quad \tau=-\frac{c}{2} \pm \frac{1}{2} \sqrt{c^{2}+4 k-4 c} .
$$

In particular, $\Gamma_{C_{1}}$ is connected and regular of degree $k-c$. The partition $V=$ $\{u\} \cup \Gamma(u) \cup \Gamma_{2}(u)$, subdivided by considering the vertices at a given distance from an arbitrary vertex $r$ of $C_{1}$, gives the scheme of figure 1 . 


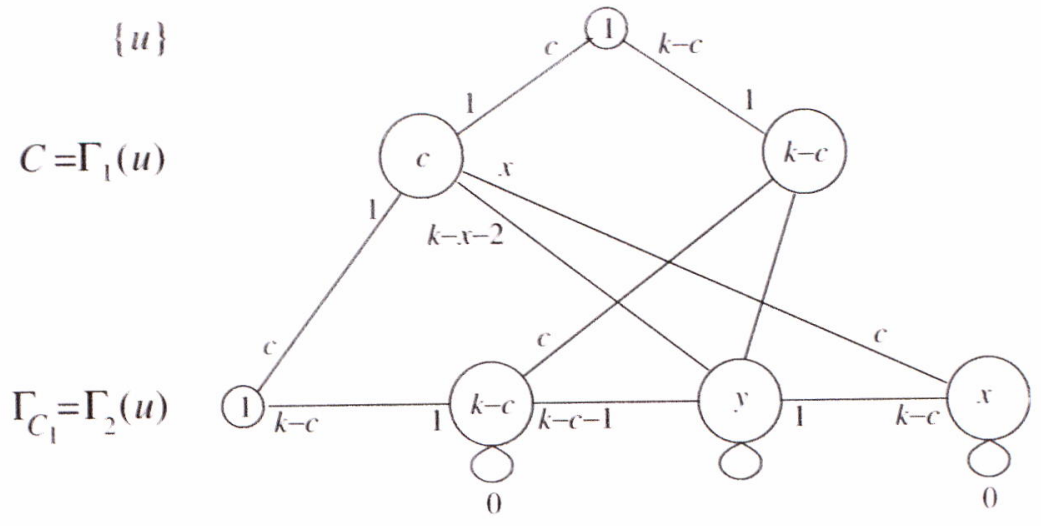

Figure 1. Intersection diagram of $\Gamma$ with $a=0$.

where we have used the combinatorial properties of $\Gamma$, as an $(n, k ; 0, c)$-strongly regular graph. From this diagram we then conclude the following.

- The number of vertices of $\left(\Gamma_{C_{1}}\right)_{3}(v)$ is

$$
x=\frac{n_{1}}{k-c+1}-1
$$

- In the graph $\Gamma_{C_{1}}$ the $x+1$ vertices of $\left(\Gamma_{C_{1}}\right)_{3}(v) \cup\{v\}$ are mutually at distance 3 since the $c$ vertices adjacent to every pair of vertices are all in $\Gamma_{1}(u)$. Therefore.

$$
\Gamma_{C_{1}} \text { is }\left(\frac{n_{1}}{k-c+1}\right) \text { - antipodal. }
$$

Notice that the number of antipodal classes is $s=k-c+1$.

Then, by (42) and Theorem 1.4, the graph $\Gamma_{C_{1}}$ is distance-regular if and only if

$$
\frac{1}{2} \sum_{i=0}^{3} \frac{\pi_{0}}{\pi_{i}}=k-c+1
$$

But. from (41), we obtain $\sum_{i=0}^{3}\left(\pi_{0} / \pi_{i}\right)=2 k$, and the above yields the equation $k=k-c+1$. Hence, $c=1$ and we have proved part (a) of Theorem 3.1 below.

Case 2 Let $i=0, j \geq 1$. Then $\Gamma_{C_{2}}=K_{n_{2}}$ and we would have $\tau=-1$, contradicting that $\Gamma$ is not trivial.

Case 3 Let $i \geq 1, j=0$. Then the first equation of system (36) yields $n_{2} \geq 2$. By $\Gamma_{C_{2}}=K_{n_{2}}$ we get $\beta=n_{2}-1>0$ and $\theta+\tau=-1$. Thus, substituting $\tau=-1-\theta$ into $(38)$ and $(40)$ the following conditions for $h$ are obtained:

$$
\begin{gathered}
Q=h^{2}-2\left(2 \theta^{2}+2 \theta-k\right) h-1-4 \theta-4 \theta^{2}+k^{2}=0, \\
k-1-2 \theta<h \leq-k+1+2 \theta+2 \theta^{2} .
\end{gathered}
$$


Furthermore, the discriminant of the second degree equation is

$$
\Delta^{\prime \prime}=\left(2 \theta^{2}+2 \theta-k+1\right)^{2}-k^{2}+2 k=4\left(\theta-x_{1}\right)\left(\theta-x_{2}\right)\left(\theta-x_{3}\right)\left(\theta-x_{4}\right) .
$$

with $x_{1}>x_{2}>0>x_{3}>x_{4}$ and

$$
x_{1.2}=-\frac{1}{2}+\frac{1}{2} \sqrt{1+(\sqrt{k} \pm \sqrt{k-2})^{2}} .
$$

Then $\Delta$ " would be non-negative for either $0 \leq \theta \leq x_{2}$ or $\theta \geq x_{1}$. In the first case.

$$
h \leq-k+1+2 \theta+2 \theta^{2} \leq-k+1+2 x_{2}+2 x_{2}^{2}=-\sqrt{k(k-2)}<0,
$$

whence this option is not possible.

Now assume that $\theta \geq x_{1}$, which is equivalent to $2 \theta+1 \geq \sqrt{1+(\sqrt{k}+\sqrt{k-2})^{2}}$. From $\theta=(1 / 2)(-1+\sqrt{1+4 k-4 c})$ we would have the inequality

$$
\sqrt{1+4 k-4 c} \geq \sqrt{1+(\sqrt{k}+\sqrt{k-2})^{2}}
$$

whence

$$
2 c \leq k+1-\sqrt{k(k-2)}=3+k-2-\sqrt{k(k-2)}=3+\sqrt{k-2}(\sqrt{k-2}-\sqrt{k})<3 .
$$

Therefore, $c=1, a=c+\theta+\tau=0$, and $\Gamma$ is a Moore graph. The polynomial $Q$ is now $Q=h^{2}-2(k-2) h+k^{2}-4 k+3$, with roots $h=k-1$ and $h=k-3$. The root $k-1$ gives $\beta=0$ and $i=0$, a contradiction. For $h=k-3$ we obtain the parameters

$$
a=0, \quad c=1, \quad r=2 k-2, \quad n_{1}=n-2 k, \quad n_{2}=2, \quad \alpha=k-2, \quad \beta=1 .
$$

The graph $\Gamma_{C_{2}}$ is $K_{2}$ and, if $V_{2}=\{u, v\}$, the independent set is $C=\Gamma(u v):=\Gamma_{1}(\{u, v\})$; that is, the set of vertices at distance one of edge $u v$. Therefore, the graph $\Gamma$ is either the Hoffman-Singleton graph with parameters $(50,7 ; 0,1)$ or the possible Moore graph with parameters $(3250,57 ; 0,1)$. Setting $k=(1 / 4)\left(t^{2}-3\right)$ with $t=5,15$, we then have

$\operatorname{sp} \Gamma_{C_{1}}=\left\{\frac{t^{2}-5}{4}>\left(\frac{t-1}{2}\right)^{(1 / 32 t)\left(t^{2}-5\right)(t-1)(t+3)}>-1^{(1 / 2)\left(r^{2}-5\right)}>\left(\frac{-t-1}{2}\right)^{(1 / 32 t)\left(t^{2}-5\right)(t+1)^{2}(t-3)}\right\}$.

For every vertex $w$ of $\Gamma_{C_{1}}$ we get

$$
n_{3}(w)=\left(k^{2}-1-r\right)-1-(k-2)-(k-2)(k-3)=2 k-4
$$

which, together with

$$
n_{1}\left(\sum_{i=0}^{3} \frac{\tilde{\pi}_{0}^{2}}{\tilde{m}_{l} \tilde{\pi}_{l}^{2}}\right)^{-1}=\frac{1}{2}\left(t^{2}-5\right)=2 k-4
$$

allows us to demonstrate part (b) of Theorem 3.1. 
Case 4 Let $i \geq 1, j \geq 1$. Then $\Gamma_{C_{2}}$ would be a strongly regular graph with eigenvalues $\beta$. $\mu_{1}=\theta+\tau$ and $\mu_{2}=\tau$. But the inequalities

$$
0 \leq \beta-\mu_{1}+\mu_{2}=\beta-\theta-\tau+\tau=\beta-\theta<0 .
$$

obtained from (14) and (25), compel us to exclude this case.

As a conclusion of the previous case-by-case study, we now have the following result. whose "if" parts are well-known (see, for instance, [3,13.18]).

THEOREM 3.1 Let $C \subset V$ be an independent set of an $(n, k ; a, c)$-strongly regular graph $\Gamma=(V, E)$. Then the following statements hold.

(a) When $C=\Gamma(u)$, the non-trivial component $\Gamma_{C_{1}}$ of the outindependent graph $\Gamma_{C}-$ or the graph induced by the vertices at distance two from $u$ - is a distance-regular graph if and only if $\Gamma$ is a Moore graph. In this case, $\Gamma_{C_{1}}$ is an antipodal $(k-1)$-cover of the complete graph $K_{k}$. with intersection array.

$$
i\left(\Gamma_{C}\right)=\{k-1, k-2,1 ; 1,1, k-1\}, \quad k \in\{7,57\} .
$$

(b) When $C=\Gamma(u v)$, with $u v \in E$, the component $\Gamma_{C_{1}}$ being different from $K_{2}$ of the outindependent graph $\Gamma_{C}-$ or the graph induced by the vertices at distance wo from $u \mathrm{w}$ - is a distance-regular graph (with diameter three) if and only if $\Gamma$ is a Moore graph. In this case, the intersection array is

$$
\iota\left(\Gamma_{C_{1}}\right)=\{k-2, k-3,2 ; 1,1, k-3\} . \quad k \in\{7,57\} .
$$

A last comment concerning case (a): From the results of Gardiner et al. [24], it was already known that if the vertices at distance two from a vertex in a strongly regular graph induce a distance-regular graph, then it is antipodal.

\section{Acknowledgment}

We sincerely acknowledge the referee for helpful comments and suggestions that led to a significant improvement of the original manuscript.

\section{References}

[1] de Caen. D.. 1998. The spectra of complementary subgraphs in a strongly regular graph. European Journal of Combinatorics. 19(5). 559-565.

[2] Haemers. W.H.. 1995. Interlacing eigenvalues and graphs. Linear Algehra and its Applications. $226-228$. 593-616.

[3] Biggs. N.L.. 1993. Algehruic Graph Theorl. 2nd Edn (Cambridge: Cambridge University Press).

[4] Jeurissen. R.H.. 1988. His graph in theirs (the Coxeter graph in that of Hoffman and Singleton). Ars Combinatoria. 25. 31-36.

[5] Fiol. M.A.. Garriga. E. and Yebra. J.L.A.. 1996. Locally pseudo-distance-regular graphs. Journal of Combinatorial Theory Series B. 68. 179-205.

[6] Fiol. M.A. and Garriga. E.. 1999. On the algebraic theory of pseudo-distance-regularity around a set. Limear Algetra and its Applications. 298(1-3). $115-141$. 
[7] Fiol, M.A. and Garriga. E. 2001. An algebraic characterization of completely regular codes in distanceregular graphs. SIAM Journal on Diserete Mathematics. 15(1). 1-13.

[8] Lancaster. P. and Tismenetsky. M.. 1985. Theory of Matrices. 2nd Edu (New York: Academic Press).

[9] Cvetkovic, D.M.. 1971. Graphs and their spectra. Livierzitet u Beogradu. Puhlikatije Elektrotechnickog Fakulteta. Serija Matematika Fiziki. 354-356. 1-50.

[10] Fiol. M.A. and Garriga. E.. 1996. From local adjacency polynomials to locally pseudo-distance-regular graphs. Journal of Combinatorial Theory Series B. 71. 162183.

[11] Fiol. M.A.. 1997. An eigenvalue characterization of antipodal distance-regular graphs. Electronic Journal of Combinatorics, 4 (1), \#R30.

$\boldsymbol{W}$.

[12] Cameron. P.J.. 1978. Strongly regular graphs. In: L.J. Beineke and R.J. Wilson (Eds) Selected Topics in Graph Theorl: Vol. (New York: Academic Press). pp. 337-360.

[13] Godsil. C.D.. 1993, Algehraic Combinatorics (London New York: Chapman and Hall).

[14] Hoffman. A.J.. 1963. On the polynomial of a graph. The American Mathematical Momth/: $70.30-36$.

[15] Bannai. E. and Ito. T.. 1984. Algehraic Combinatorics I: Association Silhemes (Menlo Park. CA: Benjamin (ummings).

[16] Lovasz. L.. 1979. On the Shamnon capacity of a graph. Institute of Electrical and Electronics Engineers Transactions on Intormation Theory. IT-25(1). 1-7.

[17] Fiol. M.A.. 1999. Eigenvalue interlacing and weight parameters of graphs. Linear Algehra Applications. 290(1-3). 275-301

[18] Brouwer. A.E.. Cohen. A.M. and Neumaier. A.. 1989. Distance-Regular Graphs (Berlin: SpringerVerlag).

[19] Chang. L.. 1959. The uniqueness and non-uniqueness of the triangular association scheme. Science Record (N.S.). 3. 604-613.

[20] Chang. L.. 1960. Association schemes of partially balanced designs with parameters $r=28 . n_{1}=12$. $n_{2}=15$ and $p_{i 1}=4$. Science Record (N.S.). 4. 12-18.

[21] Hoffman. A.J.. 1960. On the exceptional case in a characterization of the arcs of a complete graph. IBM Journal of Research and Development. 4. 487-496.

[22] Schwenk. A.J.. 1995. Eigenvalues of various subgraphs of the possible Moore graph of order 3250. In: $3 \mathrm{rd}$ Simposium on Matrix Analysis \& Applications: A Look at Recent Developments (Kalamazoo: Western Michigan University).

[23] Brouwer. A.E. and Haemers. W.H.. 1993. The Gewirtz graph: an exercise in the theory of graph spectra. European Journal of Combinalorics. 14(5). 397-407.

[24] Gardiner. A.D.. Godsil. C.D.. Hensel. A.D. and Gordon F. Royle. 1992. Second neighbourhoods of strongly regular graphs. Discrete Mahematics, 103(2). 161 170. 